\title{
EMISSION FACTORS RELATED TO VEHICLE MODAL ACTIVITY
}

\author{
O. GIUFFRE ${ }^{1}$, A. GRANA ${ }^{1}$, T. GIUFFRE ${ }^{2} \&$ R. MARINO ${ }^{1}$ \\ ${ }^{1}$ Department of Civil Environmental and Aerospace Engineering, Palermo University, Italy. \\ ${ }^{2}$ University of Kore, Enna, Italy.
}

\begin{abstract}
Polluting emissions depend on vehicle characteristics and on traffic conditions expected after the construction of a road project. Nevertheless, in the assessment of new projects, the task of road designer becomes uncertain when the reduction in polluting emissions has to be evaluated. Moreover, vehicle emissions are highly linked to modal vehicle activity, but current emission rate models do not properly predict on-road vehicle emissions produced by modal traffic events, as those occurring at intersections.

Modal emission models require the analysis of modal activity at a microscale level in order to evaluate emission factors by a single mode (idle, acceleration, deceleration, and cruise). This evaluation can be standardised with reference to the type of road, volume-to-capacity ratio and fleet composition. On the contrary, the mesoscopic level for vehicle modal activities, as it is usual in traffic analysis, will result appropriate to reach correct emission estimates.

In order to explain factors affecting polluting emissions, a research programme targeted to the development of a methodology to be applied to traffic studies has been undertaken. This paper reports the results of an exploratory analysis, based on examples of driving patterns, with the specific purpose to measure and to interpret vehicular polluting emissions in road situations different for geometric and traffic conditions. Results referred to in this paper show that, for a specific traffic condition, estimates of vehicle polluting emissions can be obtained from emission factors proper to each elementary modal activity and from proportion of time spent by vehicles in each modal activity as defined at mesoscopic level.
\end{abstract}

Keywords: emission factor, modal emissions, vehicle modal activity.

\section{INTRODUCTION}

Different from other air pollution sources (production activity of electrical energy, combustion installations, etc.), several concomitant factors work together in raising critical conditions for transport infrastructures: the slow process of renewal for the existing fleet with vehicles meeting new regulations and the growth of vehicular fleet, of the distance recovered and then of the related fuel consumptions. In this respect, non-restrictive measures concerning road traffic are widely implemented to reduce polluting emissions of vehicles, particularly those which directly influence the operational conditions of road traffic (speed, acceleration, deceleration), related to engine operation and emission factors. Nevertheless, evaluation of benefits deriving from this kind of actions is not yet integrated in current practices of impact assessment of improvement measures on road traffic and presents many aspects of uncertainty.

Emission models usually applied to plan national or regional emission inventories, as those integrated in the CORINAIR procedure, are not useful for the evaluation of vehicle emissions at microscale level. These models are designed for wide-area analysis, for which emission rates are appropriately linked to average speed. They are generally based on an aggregate representation of on-road vehicle activities and they only provide a single emission rate for each average speed level starting from emission rates obtained in standard laboratory test conditions and adjusting them on the basis of observed differences between the laboratory and field conditions. In this way, the emission models cannot reflect changes in driving behaviour and become less interesting as soon as the on-road driving cycles differ from standard laboratory tests considered for implementation. 
To obtain estimates of vehicular emissions, it is necessary to (a) use models sensible to operational conditions; (b) use models able to reflect variations in traffic conditions and geometric characteristics; (c) require accurately vehicle activity profiles (representing on-road conditions) and corresponding activity-specific emission rates. Data availability of emission estimates can become critical when is not possible to take direct measures for modal activity of vehicles and it is necessary, then, to use predictive models able to represent profiles for a single vehicle along a specific street segment or in intersection situations. Moreover, when models have to be applied in evaluating air quality effects produced by traffic improvement actions, it can be also desirable to correlate vehicle emissions to performance indicators traditionally applied to traffic analysis (e.g. the ones assumed in HCM methodologies [1]).

Current researches underline that vehicle emission rates have to be correlated to specific engine operating modes: increases in engine loads (hard accelerations, high speeds and operations on steep grades), as well as rapid decreases in engine loads (hard/long deceleration), result in high motor vehicle emission rates compared to stable engine operations [2-5]. Fraction of time spent in each operating mode by vehicles is the input data typical of modal activity. Several models have been developed by researchers in order to predict emissions as functions of specific operating modes or of engine load surrogates that represent the on-road operating conditions leading to high instantaneous emission rates (accelerations and decelerations). They are the necessary premise for valuing the effects deriving from traffic-flow improvement projects and they allow exceeding limits of models applied to traditional on-road emission inventories. Vehicle fraction of time spent in each operating mode is the typical input data of these models; as a consequence, different researches have been devoted to obtain good estimates of microscopic on-road modal vehicle activity through both numeric simulations [6-8] and field studies by means of instrumented vehicles or external observations of driving behaviour in real conditions [9-12]. In regard to disaggregation within the level of analysis, modal models can be classified in macroscopic, microscopic and mesoscopic models. Macroscopic models are based on the assumption that vehicular activity is macroscopically segregated into modal zones, such as accelerating, decelerating, cruising and idling. Deterministic algorithms are generally used to calculate queue lengths and idle time per vehicle [13]. Microscopic models are simulation models capable of producing individual vehicle profiles (vehicle operating mode) which can be used in modal emission estimation or in estimation of fuel consumption rates. Moreover, microscopic models include a wide class of models studied for specific applications ranging from micro-area level to regional one [14-16]. At last, mesoscopic models combine properties of microscopic and macroscopic models (e.g. assuming the individual vehicle as unit of traffic flow, but considering its movement governed by the average speed on the travel link). In these models, travel simulation generally takes place on an aggregate level and it does not consider dynamic relationships between speed and volume [17]. It must be noted that simulation models can provide estimates of modal activity unsuitable as input data to emission models. Also, for microscopic models, limitations exist in their ability to describe accurately specific velocity-acceleration combinations that are crucial when modal emission models have to be used $[2,9]$.

In light of this, research efforts were directed towards the determination of on-road vehicle activity as representative as possible of real-world conditions. Moreover, the reliability of the vehicle emission estimates is highly conditioned by local variables (especially by fleet compositions, traffic conditions typical of the examined road environment, users' behaviour, etc.), so that experience gained in a specific context cannot be directly transferred to another situation.

Starting from these considerations, a specific research programme was implemented to develop a decision-making tool for evaluating the effectiveness of road-network projects, on the basis of effects on the efficiency of road traffic and on risk related to polluting emissions. Considering the 
close relationship between the emission phenomenon and instantaneous characteristics of road traffic, a specific research has been devoted to model polluting emissions from vehicles in different road conditions for geometric and traffic features.

This paper reports the results of a preliminary survey carried out with the objective both to examine the influence of modal activity on emission levels and to identify the most appropriate scale (microscopic and/or mesoscopic) for evaluating effects of traffic improvements on the emission phenomenon.

\section{BASIC ASSUMPTIONS AND DERIVATION OF EMISSION FUNCTIONS}

In order to pursue the research objectives, a modal emission modelling approach has been proposed starting from the method developed by Dowling et al. [18], as referred to in the NCHRP Report 535. This method represents an updated synthesis between numeric micro-simulation approaches and experimental procedures that are based on direct observations of the modal activity.

The first step of the research required to calculate total vehicle-hours (VHT) by vehicle operating mode (i.e. acceleration, cruise, deceleration and idle), defined by a speed/acceleration category. The estimates of the vehicle activity have been then used with modal emission factors to produce the emission estimates.

Vehicle modal activity tables for arterial sections produced by CORSIM model [18] have been used to compute the proportion of vehicle hours spent on a network link $i$ in a mode of operation $j$ as a function of the link type and of the volume-to-capacity ratio $(v / c)$. Such speed and acceleration frequency distributions (SAFDs) show the splitting up of total time spent both over the speed/freeflow speed ratio and over the acceleration/deceleration categories. They represent proportions of time spent in each mode of operation for the considered facility, but normalised for free-flow speed, and permit to account for differences in road design characteristics.

Figure 1 shows SAFDs as issued by the NCHRP Report 535; in this figure, Spd/FreSpd is used as an acronym of speed/free-flow speed ratio. Facility types and/or traffic management that are different from those considered in the above-mentioned report need to create proper SAFDs through micro-simulation tools.

The next step of the research considered running exhaust emission rates $q_{R}(i, j)$ for specified SAFDs, as those produced by the Comprehensive Modal Emission Model (CMEM) in units of grams per second. Total running exhaust emission for a non-specific pollutant $R$ will be in the form:

$$
E_{R}=\sum_{i j} q_{R}(i, j) \times v(i, j)
$$

where $v(i, j)$ identifies VHT at speed $i$ and at acceleration $j$, which is the joint speed-acceleration frequency distribution (cumulative vehicle seconds) for the $i$ th speed category and $j$ th acceleration category.

As it is well known, CMEM considers carbon monoxide (CO), hydrocarbon (HC) and oxides of nitrogen $\left(\mathrm{NO}_{x}\right)$; it does not provide emission rates for heavy-duty vehicles and it is calibrated on a composite light-duty vehicle representative of US fleet. Figure 2 shows, as an example, CMEM light-duty vehicle $\mathrm{CO}$ emission rates for each speed and acceleration category.

The examined phenomenon was then represented through the functions of emission that, for each pollutant, link the emission rate with specific operational conditions.

To characterise operational conditions of arterials, the ratio of average speed over free-flow speed has been used; this variable is determined considering that, for an assigned speed-acceleration frequency distribution $v(i, j)$, it must result: average speed $=\sum_{i}\left[s_{i} \cdot \sum_{j} v(i, j)\right]$, where $s_{i}$ represents the 

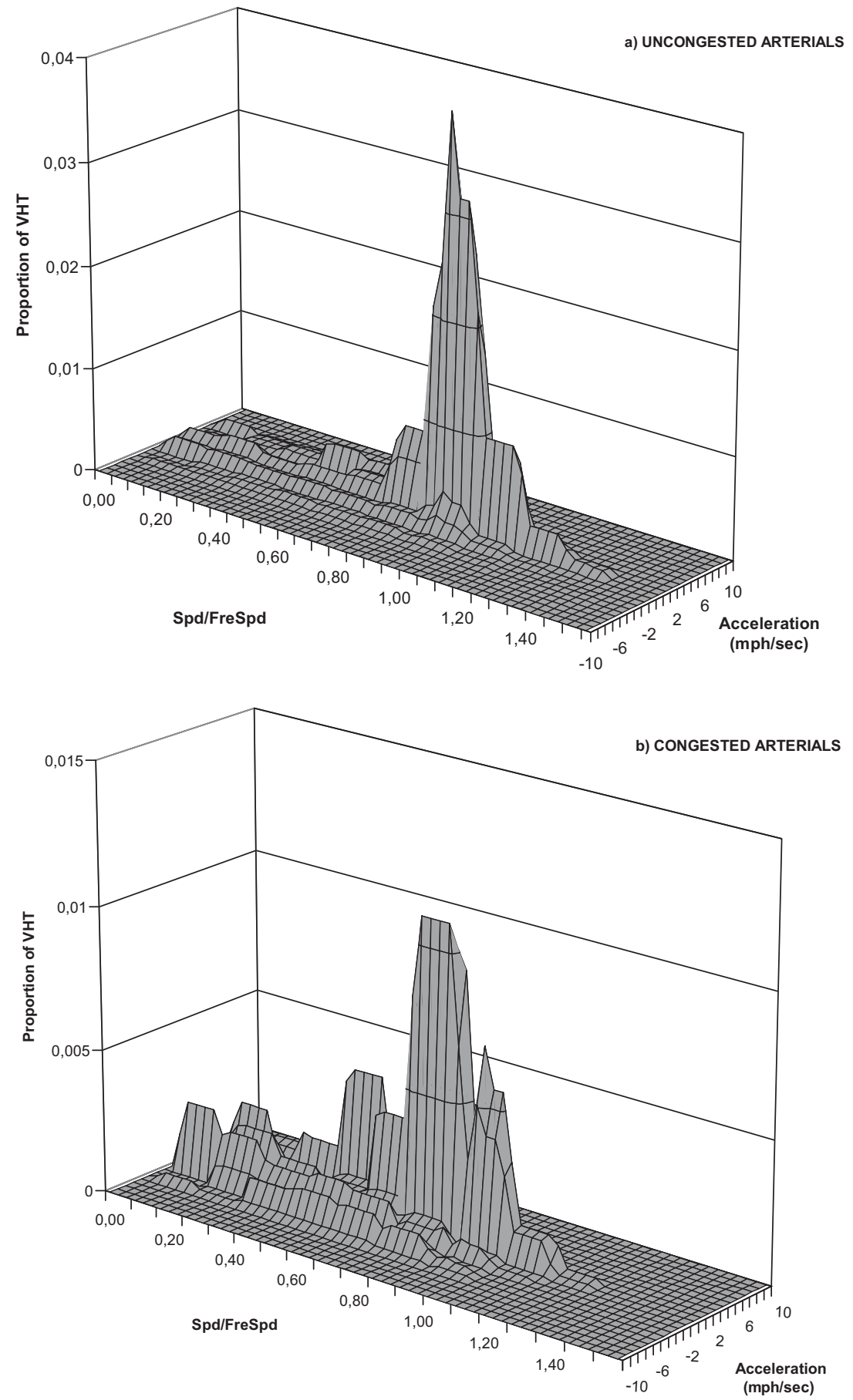

Figure 1: Vehicle modal activity for (a) uncongested arterials (proportion of time spent in 0/0 speed/ acceleration category (0.2006) is not represented) and (b) congested arterials (proportion of time spent in $0 / 0$ speed/acceleration category (0.5317) is not represented). 


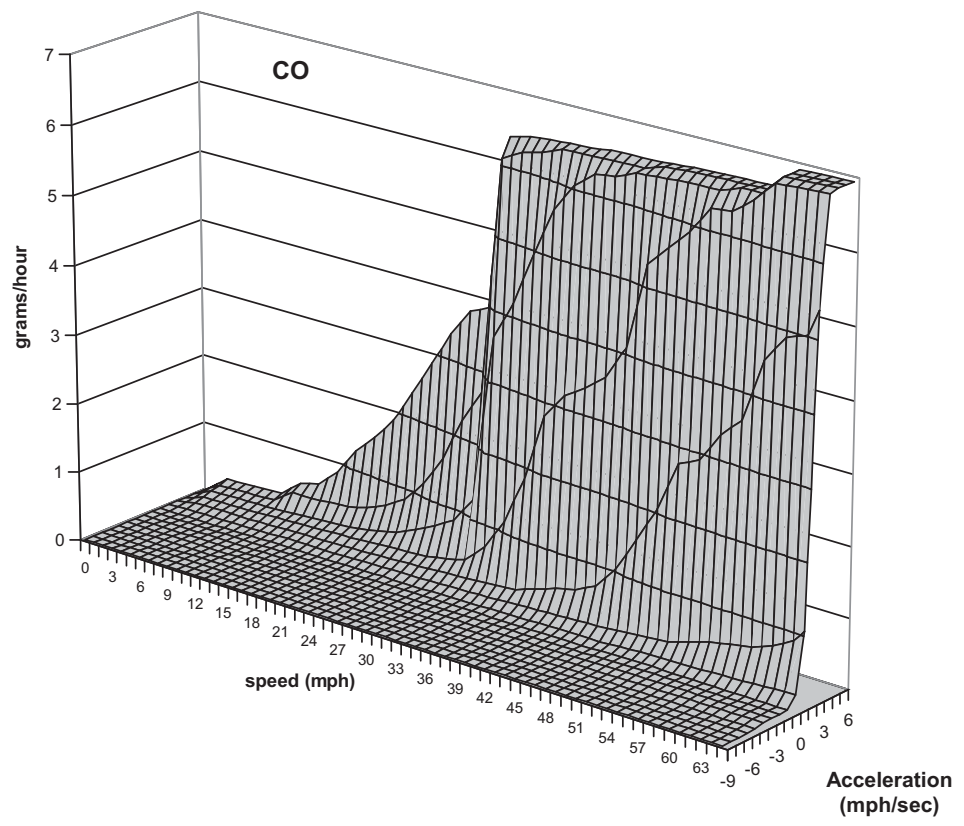

Figure 2: CMEM light-duty vehicle CO emission rates.

value of the $i$ th speed category. Intermediate SAFDs different from the uncongested and the congested conditions (see NCHRP Report 535) have to be also derived.

Only the uncongested and congested conditions will be considered for arterials; the corresponding average speed/free-flow speed ratio is 0.28 (congested condition) and 0.65 (uncongested condition).

Figures 3a-5a show emission rates expressed in grams per unit of VHT calculated for each pollutant and for different road design elements of arterials; by way of example, indeed, free-flow speed ranging from 40 to $60 \mathrm{~km} / \mathrm{h}$ has been considered as representative with reference to the type of road under examination. Emission rates in grams for VHT are clearly not related to the permanence time of vehicles on arterials; for a single vehicle, this time increases as traffic conditions approach the congestion.

Figures $3 b-5 b$ show emission rates expressed in grams per vehicle kilomtre for each pollutant and for different values of free-flow speed. This representation was made to highlight the influence of running time (i.e. total travel time less time staying in idle) on the emission phenomenon. It can be noted that regardless of the type of pollutant, emission rates are exactly the same in the uncongested conditions when free-flow speed reaches or goes over $50 \mathrm{~km} / \mathrm{h}$; the fact reflects the opposite effect of the free-flow speed on the time spent on the road and on the emission factor (in g/VHT). Emission rates as above computed represent the weighted mean of elements belonging to the CMEM matrix with regard to the frequency of the correspondent bin in the proper speed/acceleration frequency distributions.

Changes in road characteristics and in operational conditions (from uncongested to congested) produce variations in emission rates only depending on modifications of the vehicular modal activity; these changes result from a different distribution between delay events (i.e. conditions that cause the vehicle to decelerate, to stay in idle, to accelerate) and cruise events, during which the vehicle is moving almost uniformly at the cruising speed. 

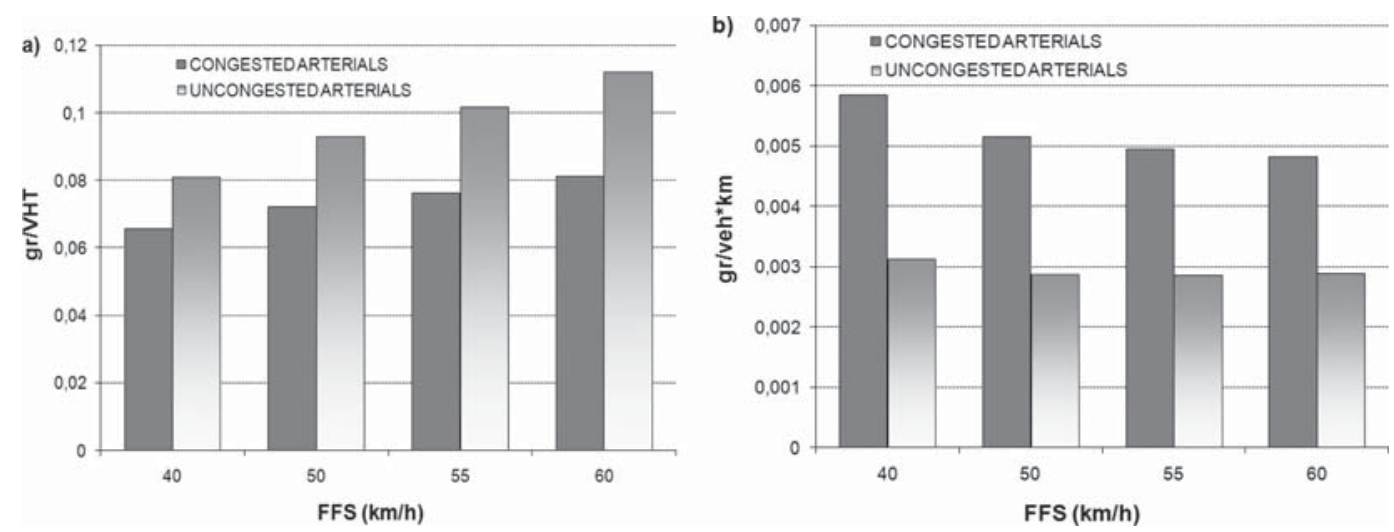

Figure 3: CO emission functions in $[\mathrm{g} / \mathrm{VHT}]$ and in $[\mathrm{g} / \mathrm{veh} \times \mathrm{km}]$.
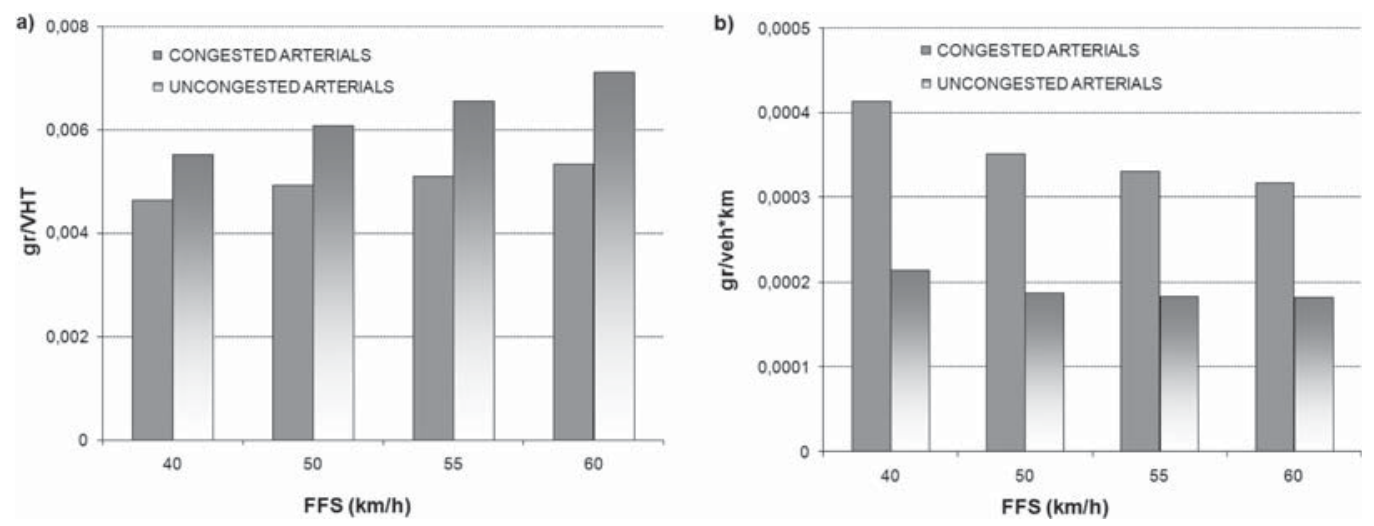

Figure 4: HC emission functions in $[\mathrm{g} / \mathrm{VHT}]$ and in $[\mathrm{g} / \mathrm{veh} \times \mathrm{km}]$.

a)

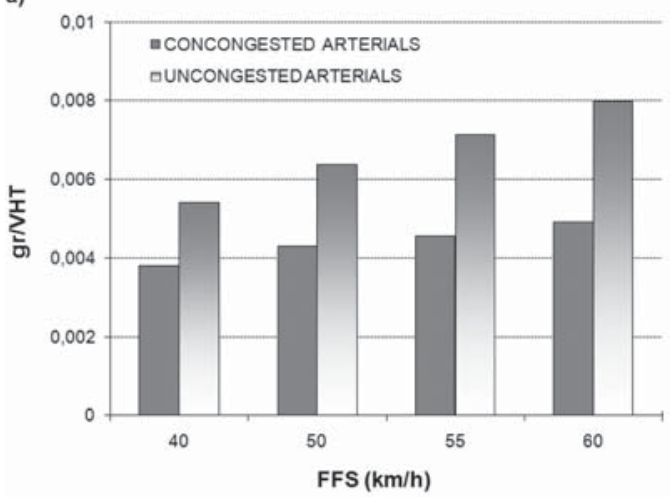

b)

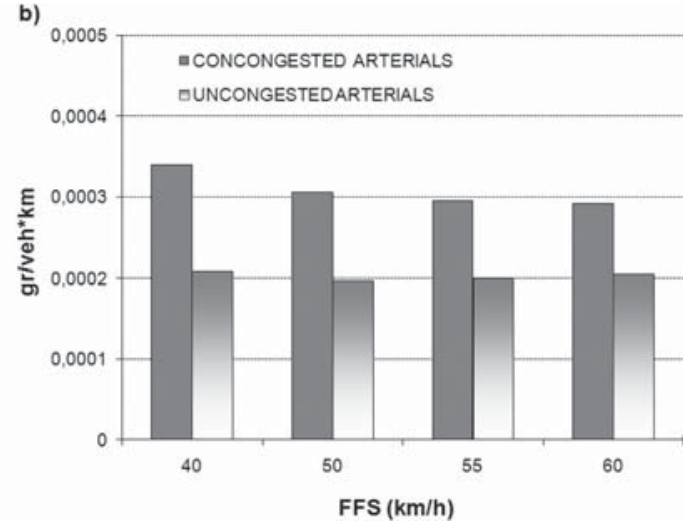

Figure 5: $\mathrm{NO}_{X}$ emission functions in [g/VHT] and in $[\mathrm{g} / \mathrm{veh} \times \mathrm{km}]$. 


\section{EMISSION FACTORS RELATED TO MODAL ACTIVITY}

The next step of the proposed approach concerned the study of the relation between emission factors and elementary traffic events; for this purpose, partial aggregations of SAFDs have been used as input to CMEM model to predict emission factors for each elementary traffic event. In order to recognise each elementary modal activity, a criterion has been proposed (see Table 1) both examining results of direct observations of driving patterns, as referred to in [12], and considering SAFDs related to different level-of-services [19].

By the light of the stated criterion, analysis of SAFDs for arterials in different traffic conditions (uncongested/congested) allowed to identify the proportion of time spent by vehicles in each modal activity (see Fig. 6).

To obtain the contribution of each mode to the emission phenomenon through the CMEM model, calculations were developed for congested and uncongested arterials. Table 2 shows the emission factors only when FFS is equal to $55 \mathrm{~km} / \mathrm{h}$.

Results show that the contribution given by cruise mode in uncongested/congested conditions for each pollutant is substantial. Acceleration and deceleration modes differ from idle and cruise modes and give a nearly equal contribution on the total emission regardless of traffic conditions (uncongested/congested).

It has to be noted that results in Table 2 reflect the modal activity distributions considered in the NCHRP Report 535. They are strictly applicable when the same conditions occur and, apart from the methodological value, the practical interest can be limited. Nevertheless, a good generalisation of the proposed approach can be obtained considering that the contribution of each modal activity on

Table 1: Definition criterion for elementary traffic event.

\begin{tabular}{lccc}
\hline Modal activity & Deceleration & Speed & Acceleration \\
\hline Idle & $\geq-1 \mathrm{mph} / \mathrm{sec}$ & $\leq 3 \mathrm{mph}$ & $\leq 1 \mathrm{mph} / \mathrm{sec}$ \\
Acceleration & - & All & $\geq 2 \mathrm{mph} / \mathrm{sec}$ \\
Deceleration & $\leq-2 \mathrm{mph} / \mathrm{sec}$ & All & - \\
Cruise & $\geq-1 \mathrm{mph} / \mathrm{sec}$ & $>3 \mathrm{mph}$ & $\leq 1 \mathrm{mph} / \mathrm{sec}$ \\
\hline
\end{tabular}

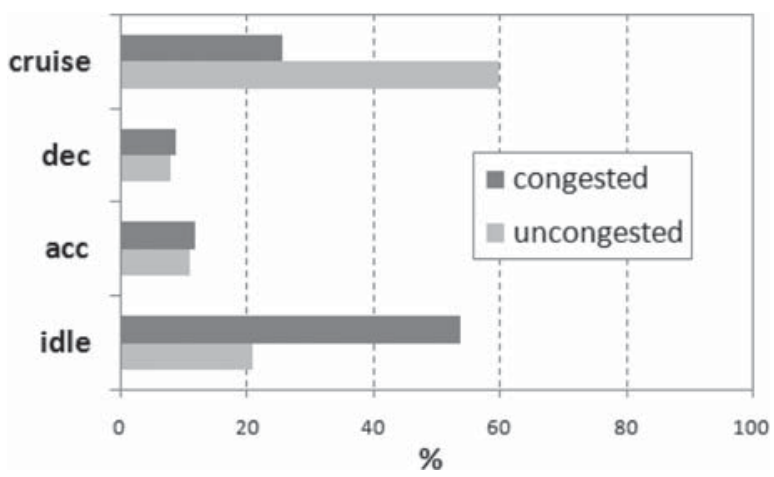

Figure 6: Percentages $(\%)$ of time spent by modal event $(\mathrm{FFS}=55 \mathrm{~km} / \mathrm{h})$. 
Table 2: Emissions by modal activity for arterial (uncongested/congested) conditions [g/VHT].

\begin{tabular}{llccc}
\hline Modal activity & Condition & $\mathrm{CO}(\mathrm{g} / \mathrm{VHT})$ & $\mathrm{HC}(\mathrm{g} / \mathrm{VHT})$ & $\mathrm{NO}_{x}(\mathrm{~g} / \mathrm{VHT})$ \\
\hline \multirow{2}{*}{ Idle } & Uncongested & 0.0077 & 0.0006 & 0.0003 \\
\multirow{3}{*}{ Acceleration } & Congested & 0.0198 & 0.0017 & 0.0007 \\
& Uncongested & 0.0268 & 0.0015 & 0.0019 \\
Deceleration & Congested & 0.0288 & 0.0015 & 0.002 \\
& Uncongested & 0.0034 & 0.0003 & 0.0001 \\
Cruise & Congested & 0.0035 & 0.0003 & 0.0001 \\
& Uncongested & 0.0638 & 0.0042 & 0.0048 \\
& Congested & 0.0241 & 0.0016 & 0.0018 \\
\hline
\end{tabular}

the total emission derives from two concomitant factors: (i) from particular distribution of speed and acceleration categories characterising each mode and from variations due to changed operational conditions and (ii) from the time globally spent in each modal activity, varying as well with operational conditions.

In the case of arterials, the time distribution by modal activity is influenced by delay events deriving from intersection type and spacing. To highlight the weight of the time spent in a single modal activity, we can put eqn (1) in the following manner:

$$
\begin{aligned}
E_{R}=\sum_{i j} q_{R}(i, j) \cdot v(i, j) & =\sum_{m} \sum_{i, j} q_{R}^{m}(i, j) \cdot v^{m}(i, j) \\
& =\sum_{m} \tau^{m} \cdot \sum_{i, j} \frac{q_{R}^{m}(i, j) \cdot v^{m}(i, j)}{\tau^{m}}
\end{aligned}
$$

where $\tau^{m}$ is the proportion of the time spent in a specific mode;

$$
\sum_{i, j} \frac{q_{R}^{m}(i, j) \cdot v^{m}(i, j)}{\tau^{m}}
$$

represents the emission linked to $m$ mode, i.e. the quantity of pollutant that would be emitted if vehicle spends all its time in a single mode (single mode emission level).

Table 3 shows an example of results obtained for the case of arterials before examined, when the effect of the free-flow speed on the time spent on the road is disregard.

Single modal emissions for arterials in grams per unit of VHT show, regardless of the type of considered pollutant, light differences in single mode emissions for different operational conditions. It can be concluded that the total emission level derives mainly from proportion of time spent in each operational mode and from the emission factor by mode. The variations characterising at microscopic level the distribution of speed and acceleration categories can be marginal if they are considered within the same modal activity both in general and with regard to the imprecision by which vehicle emission estimates can be obtained. 
Table 3: Single modal emissions for arterials $(\mathrm{FFS}=55 \mathrm{~km} / \mathrm{h}$ ).

\begin{tabular}{llccc}
\hline Modal activity & Condition & $\mathrm{CO}(\mathrm{g} / \mathrm{VHT})$ & $\mathrm{HC}(\mathrm{g} / \mathrm{VHT})$ & $\mathrm{NO}_{x}(\mathrm{~g} / \mathrm{VHT})$ \\
\hline \multirow{2}{*}{ Idle } & Uncongested & 36.91 & 3.09 & 1.31 \\
& Congested & 36.80 & 3.08 & 1.30 \\
Acceleration & Uncongested & 249.10 & 13.59 & 17.62 \\
& Congested & 245.18 & 13.09 & 16.83 \\
\multirow{2}{*}{ Deceleration } & Uncongested & 42.57 & 3.49 & 1.48 \\
& Congested & 40.30 & 3.40 & 1.29 \\
Cruise & Uncongested & 105.88 & 6.92 & 8.05 \\
& Congested & 93.95 & 6.23 & 6.84 \\
\hline
\end{tabular}

\section{HOW TO DERIVE THE PROPORTION OF TIME SPENT BY MODE AT URBAN ARTERIALS}

The previous discussion on emission factors related with traffic events shows that, without any loss of accuracy, it is possible to reach estimates of polluting emissions made by vehicles in a determined traffic condition, from emission factors proper of each mode (e.g. through single mode emission factor) and from the distribution of the total travel time in modal classes (idle, acceleration, deceleration, cruise) defined at mesoscopic level. In practical applications, modal activity derived from traffic study methodologies can be thus considered a valid alternative to traffic micro-simulation models or to driving patterns derived from floating instrumented cars or other experimental devices.

In order to give an example of the proposed approach, the following considerations will show how to derive the time spent by vehicles in each modal activity.

Total time that vehicles spend on a segment of an urban arterial can be split up into two components: the running time and the control delay at signalised intersections.

The first represents the portion of travel time during which a vehicle is in motion; it can be estimated using FFS, urban street classification and arterial segment length. Several factors can influence the actual running time; these are also assumed to affect FFS. The running time captures the effect of interactions among vehicles due to differences in driving speeds or to downstream vehicles accelerating from a stop and having not yet reached FFS. By the modal activity point-of-view, the running time is representative of the time spent in the cruise mode. On the contrary, the control delay at intersections is the component of delay that results when a control signal causes vehicles to reduce speed or to stop; it can be considered as the additional time spent decelerating-stopping-accelerating at intersection approaches. This time includes, by the modal activity point of view, deceleration, idle and acceleration modes and the distribution among elementary components of control delay must be estimated.

A way to assay these estimates is to consider the control delay per vehicle as the sum of (i) the time-in-queue per vehicle $\left(d_{\mathrm{vq}}\right)$ and (ii) the time spent in acceleration/deceleration modes $\left(d_{\mathrm{ad}}\right)$.

The average time-in-queue per vehicle arriving during the survey period can be measured on field and computed by:

$$
d_{\mathrm{vq}}=f \times\left(I_{\mathrm{s}} \times \frac{\sum V_{\mathrm{iq}}}{V_{\text {tot }}}\right)
$$


where

$I_{\mathrm{s}}=$ interval among vehicle-in-queue counts (s);

$\sum^{\mathrm{s}} V_{\mathrm{iq}}=$ sum of vehicle-in-queue counts (veh), i.e. vehicles included in the queue of stopping vehicles and have not yet exited the intersection;

$V_{\text {tot }}=$ total number of vehicles arriving during the survey period (veh);

$f=$ empirical adjustment factor; on this regard, HCM 2000 proposes $f$ equal to 0.9 .

The component $d_{\text {ad }}$ is equal to the fraction of vehicles stopping $\left(V_{\text {stop }}\right)$, corrected by an acceleration-deceleration delay correction factor $\mathrm{CF}$ appropriate to the lane group free-flow speed and to the average number of vehicles stopping per lane in each cycle (see Exhibit A16-2 in HCM 2000), as follows:

$$
d_{\mathrm{ad}}=C F \cdot \frac{V_{\text {stop }}}{V_{\text {tot }}}
$$

where

$V_{\text {stop }}=$ number of vehicles with stop;

$V_{\text {tot }}=$ total number of vehicles arriving during the survey period (veh).

The number of vehicles with stop can be evaluated based on the arrival process applicable to the examined case and on the control cycle characteristics. In the case of uniform arrival process, the evaluation of the total number of vehicles with stop $\left(V_{\text {stop }}\right)$ can be reached through the continuum model. According to this model, the total number of vehicles with stop $\left(V_{\text {stop }}\right)$ will be the sum of vehicles arriving during the red period forced to a stop $\left(V_{\text {stop }}^{\prime}\right)$ and of vehicles arriving during the clearance time $\left(V^{\prime \prime}{ }_{\text {stop }}\right)$. So, in the analysis period $T$, the above addends are, respectively,

$$
V_{\text {stop }}^{\prime}=Q_{k} \cdot r_{k} \cdot\left(\frac{T}{c_{k}}\right)
$$

where

$Q_{k}=$ flow rate at $k$ th intersection along the arterial (vph);

$r_{k}=$ effective red period (s);

$c_{k}=$ cycle length $(\mathrm{s})$.

$$
V_{\text {stop }}^{\prime \prime}=Q_{k} \cdot g_{q k} \cdot\left(\frac{T}{c_{k}}\right)
$$

where

$$
g_{q k}=\frac{\left(Q_{k} \cdot r_{k}\right)}{\left(S_{k}-Q_{k}\right)}=\text { the clearance time, i.e. during the time the queue is discharging at the satura- }
$$

tion rate $S_{k}$ until the queue is dissipated, and $S_{k}=$ saturation flow (vph).

\section{CONCLUSIONS}

The exploratory analysis reported in this paper allowed to derive through vehicle emissions models the relative weight of emission factors with relation to traffic events; it highlighted, as well, the importance assumed by the estimate of the travel time and of its modal distribution for any practical application. In the case of the arterials, regardless of the considered pollutant, it has been 
demonstrated that the level of total emissions derives primarily from the percentage of time spent in each mode (as it can be evaluated by current traffic analysis tools) and from the level of emissions related to it; it stresses the fact that changes characterising at the microscopic level the distribution of speed and acceleration categories can be quite marginal when they are considered within the same modal activity.

Results also suggest that the evaluation of emission levels, for each considered pollutant, can be mainly standardised through the type of road, volume-to-capacity ratio and fleet composition; it cannot deny the importance of analysis of modal activity at microscale level (as required by modal emission models) to derive reliable emission factors by single mode. It follows that mesoscopic level for evaluating vehicle modal activities, as it is usual in traffic analysis, may be appropriate to reach accurate emission estimates.

In order to develop a methodology suitable to be adopted for road-network project assessments, the above considerations allow, as well highlight some directions for future investigations. First, it will be necessary to deduce real driving patterns suitable to reflect different roads, traffic conditions and drivers' characteristics related to the area of interest. Moreover, to derive SAFDs by mode, it will be important to analyse vehicle modal activity on segments and at intersections. It will also be necessary to acquire vehicle data counts because emission factors for each pollutant can vary greatly among vehicular classes. Then, particular attention will be addressed to evaluate emission factors by speed and acceleration categories, reflecting a fleet composition different from the one considered by CMEM model, so they will be applicable to regional fleet characteristics.

\section{REFERENCES}

[1] HCM 2000, TRB, Special Report No 209, Transportation Research Board-National Research Council, 2000.

[2] Yu, L., Remote vehicle exhaust emission sensing for traffic simulation and optimization models. Transportation Research D, 3(5), pp. 337-347, 1998.

[3] Barth, M., An, F., Younglove, T., Scora, G., Levine, C., Ross, M., Arbor, A. \& Wenzel, T., NCHRP Project 25-11 Final Report: The Development of a Comprehensive Modal Emissions Model, National Cooperative Highway Research Program-Transportation Research Board, National Research Council, April 2000.

[4] Younglove, T., Scora G. \& Barth M., Designing on-road vehicle test programs for the development of effective vehicle emission models. Transportation Research Record: Journal of the Transportation Research Board, No. 1941, TRB-National Research Council, Washington, D.C., pp. 51-59, 2005.

[5] Barth, M., An, F., Younglove, T., Scora, G., Levine, C., Ross, M., Arbor, A. \& Wenzel, T., Development of a Comprehensive Modal Emissions Model. TRB's National Cooperative Highway Research Program (NCHRP), Web-Only Document 122, 2009.

[6] Aycin, M.F. \& Benekohal, R.F., Comparison of car-following models for simulation. 78th Annual Meeting of the Transportation Research Board, Washington D.C., 1999.

[7] Marsden, G., Bell, M., \& Reynolds, S., Towards a real-time microscopic emissions model. Transportation Research Part D: Transport and Environment, 6(1), pp. 37-60, 2001.

[8] Bham G.H. \& Benekohal, R.F., A high fidelity traffic simulation model based on cellular automata and car-following concepts. Transportation Research Part C: Emerging Technologies, 12(1), pp. 1-32, 2004.

[9] Chundury, S. \& Wolshon, B., Evaluation of the CORSIM car following model using GPS field data. 79th Annual Meeting of the Transportation Research Board, 2000. 
[10] Prakash R. \& Takashi N., Advances in microscopic traffic data collection using instrumented vehicles. Traffic engineering \& control, 47(4), pp. 147-151, 2006.

[11] Hallmark, S.L., Randall, G. \& Formunung, I., Characterizing on-road variables that affect passenger vehicle modal operation. Transportation Research Part D, 7, pp. 81-98, 2002.

[12] Frey, H.C., Rouphail, N.M., Colyar, J.D. \& Unal, A., Vehicle emissions and traffic measures: exploratory analysis of field observations at signalized arterials. 80th Transportation Research Board Annual Meeting, Washington D.C, pp. 1-27, 2001.

[13] Al-Deek, H.M., Wayson, R.L., Cooper, C.D., Keely, D., Traynelis, R., Liu, P.S., Malone, L.C. \& Datz, A., Queueing algorithm for calculating idling emissions in FLINT: the FLorida INTersection Air Quality Model. Transportation Research Record, 1587, pp. 128-136, 2007.

[14] Dion, F., Van Aerde, M. \& Rahka, H., Mesoscopic fuel consumption and vehicle emission rate estimation as a function of average speed and number of stops. 79th Annual Meeting, Washington DC, Paper 00-1134, 2000.

[15] Rakha, H., Van Aerde, M., Ahn, K. \& Trani, A.A., Requirements for evaluating traffic signal control impacts on energy and emissions based on instantaneous speed and acceleration measurements. Transportation Research Record, 1738, pp. 56-67, 2007.

[16] Fellendorf, M. \& Vortisch, P., Integrated modeling of transport demand, route choice, traffic flow and traffic emissions. 79th Annual Meeting, Washington DC, 2000.

[17] Florian, M., Mahut, M., \& Tremblay, N., Application of a Simulation-Based Dynamic Traffic Assignment Model. In Simulation Approaches in Transportation Analysis, Vol. 31, Springer, US, pp. 1-22, 2005.

[18] Dowling, R., Ireson R., Skabardonis, A., Gillen, D. \& Stopher, P., NCHRP Report 535: Predicting Air Quality Effects of Traffic-Flow Improvements: Final Report and User's Guide, Transportation Research Board-National Research Council, 2005.

[19] Carlson, T. \& Austin T., Development of Speed Correction Cycles - Report No. SR97-04-01, U.S. Environmental Protection Agency, Assessment and Modelling Division, Sierra Research Inc., California, 1997. 